\title{
MATHEMATICAL MODEL OF DISSIPATIVE PARAMETRIC VIBRATIONS OF FLEXIBLE PLATES WITH NONHOMOGENEOUS BOUNDARY CONDITIONS
}

\author{
J. AWREJCEWICZ, V. A. KRYSKO, AND T. MOLDENKOVA
}

Received 31 August 2005; Accepted 11 September 2005

In this work, parametric vibrations of flexible squared plates with changeable boundary conditions along their contours are studied. The known T. von Kármán equations serve as a mathematical model. This continuous system is reduced to a discrete one through the method of finite approximations of $O\left(h^{4}\right)$ order, which is solved further by the fourthorder Runge-Kutta technique. New scenarios of transition from harmonic to chaotic vibrations are reported.

Copyright (c) $2006 \mathrm{~J}$. Awrejcewicz et al. This is an open access article distributed under the Creative Commons Attribution License, which permits unrestricted use, distribution, and reproduction in any medium, provided the original work is properly cited.

\section{Introduction}

It is well known that plates (shells) are members of various engineering structures, and it is highly expected that their dynamical behavior should be fully understood.

On the other hand, there are many publications devoted to building low-dimensional approximating systems that capture the key features exhibited by parametric vibrations of flexible plates. The main idea of these approaches is oriented to the projection of the investigated partial differential equation(s) into the most energetic modes, obtained either through the Bubnov-Galerkin projection or via proper orthogonal decomposition. For instance, the method of multiple scales has been used by Abe et al. [1] to analyze the two modes response of simply supported laminated plates subjected to harmonic excitation. The multiple scales technique is applied to analyze subharmonic traveling waves of thin, axisymmetric, and geometrically nonlinear circular plates [21].

The Bubnov-Galerkin method is used in [22] to derive the averaged equations from the von Kármán equations and to analyze both local and global bifurcations of a parametrically and externally excited simply supported rectangular thin plate. When a plate is thin, its material is elastic and Hook's and Kirchhoff's hypotheses are valid, then the mathematical model is governed by the von Kármán equations. 
A spirit of this work relies on a properly chosen and testified algorithm of solution to the nonlinear partial differential equations (PDEs). Note that spatial- and time-dependent structural members can exhibit very fascinating dynamics, and without any doubt more nonlinear phenomena can be found in comparison to lumped systems, that is, modeled by ordinary differential equations. Among others, we mention different kinds of internal and external resonances, exchange of energy depending on the modes of internal and external resonances, coupled mode response when only one is excited, standing and traveling waves, periodic, quasiperiodic, and chaotic behaviors, various bifurcations, steady state, transitional, spatial, and temporal chaoses, and so on.

Generally, in order to study thin flexible plates governed by the von Kármán equations, one deals either with asymptotical (semiasymptotical) or purely numerical approaches (or combined asymptotical-numerical ones). Although there are many advantages of the asymptotical techniques [5], there also are some disadvantages [2, 3].

Since we decided to consider the von Kármán equations, then the best approximation is that associated with either a difference scheme or with the usually applied BubnovGalerkin procedure. Note that very often the governing partial differential equations affecting the behavior of continuous systems are transformed to a nonlinear set of ordinary differential equations using the Bubnov-Galerkin procedure in most studies. However, the latter approximation depends very much on the number of the Fourier series terms used. The main drawback always concerns the open question: how the obtained averaged equations (multidegree-of-freedom models) approximate the initial infinite dimensional object.

As we deal further with the von Kármán equations, it should be noticed that the purely mathematical problems associated with this equation have been recently addressed $[7,15$, $16,20,22]$.

On the other hand, as recent results show, the earlier-mentioned "rough" approximations of plate dynamics through a few modes approximation may lead even to improper results $[6,8,9,13,14]$.

In addition, it often happens that plates used in many engineering structures, such as aircraft fuselages, turbine discs, and reinforced aircraft bosses and wings, are characterized by nonhomogenous boundary conditions.

The present research belongs to a large project concerning mechanical objects modeled by the von Kármán equations (see the series of references $[9,10,12-14,17,18]$ ). In the mentioned references, complex vibrations and bifurcations of plates treated as systems studied with the use of classical tools associated with theoretical background of deterministic chaos, and both the Bubnov-Galerkin methods with high-order approximations and finite difference methods with approximations $O\left(h^{4}\right)$ and $O\left(h^{2}\right)$ approaches are applied and the obtained results are compared. Many new examples of nonlinear phenomena have been illustrated and discussed.

On the contrary, investigations of parametric vibrations of flexible dissipative systems represented by rectangular plates with nonconstant boundary conditions along their contours have not been done neither by other researchers nor in the earlier-mentioned references. We aim to fill the gap occurring in the research devoted to this subject by using the method of finite approximations of the $O\left(h^{4}\right)$ order, which has no drawbacks associated with the application of a few modes approximations. 


\section{Fundamental hypotheses and the governing equations}

The known equations governing the dynamics of flexible isotropic plates are taken as a mathematical model. A plate material is elastic, and both Hook's and Kirchhoff's hypotheses are valid. The plate is thin and the hypothesis on an average deflection holds. The known von Kármán equations satisfy the listed hypotheses, which have the form

$$
\begin{gathered}
\frac{\partial^{2} w}{\partial t^{2}}+\varepsilon \frac{\partial w}{\partial t}=-\frac{1}{12\left(1-v^{2}\right)} \nabla^{2} \nabla^{2} w+L(w, F)-P_{x} \frac{\partial^{2} w}{\partial x^{2}}+q, \\
\nabla^{2} \nabla^{2} F=-\frac{1}{2} L(w, w),
\end{gathered}
$$

where $w(x, y, t)$ is the deflection function along the $z$ coordinate oriented toward the Earth center and $F(x, y, t)$ is Airy's function.

Equation (2.1) is already transformed to the nondimensional form (variables with bars are nondimensional and they are omitted in (2.1)).

The following relations between dimensional and nondimensional quantities hold:

$$
\begin{gathered}
x=a \bar{x}, \quad y=b \bar{y}, \quad w=2 H \bar{w}, \quad \lambda=\frac{a}{b}, \\
t=t_{0} \bar{t}, \quad \varepsilon=(2 H) \bar{\varepsilon}, \quad P_{x}=\frac{E(2 H)^{3}}{b^{2}} \bar{P}_{x}, \\
F=E(2 H)^{3} \bar{F}, \quad q=\frac{E(2 H)^{4}}{a^{2} b^{2}} \bar{q} .
\end{gathered}
$$

The following notations are used: $P_{x}(y, t)$, longitudinal load along $O x$ axis; $2 H$, plate thickness, $a, b$, plate dimensions; $\varepsilon$, damping; $E$, Young modulus; $v$, Poisson coefficient (during calculations $\nu=0.3$ has been taken); and a coordinate system $O x y$ is situated in a low-left corner of the plate. The plate volume is $G \in\{x, y \mid 0 \leq x \leq 1,0 \leq y \leq 1\}$, $-H \leq z \leq H$. Airy's function satisfies the following relations:

$$
T_{x x}=\frac{\partial^{2} F}{\partial y^{2}}-P_{x}, \quad T_{y y}=\frac{\partial^{2} F}{\partial x}, \quad T_{x y}=\frac{\partial^{2} F}{\partial x \partial y},
$$

where $T_{x x}, T_{y y}$, and $T_{x y}$ are the stresses occurring in the middle plate surface, and $L(w, f)$ is the known nonlinear operator.

Note that the algorithm used to reduce the PDEs (2.1) with the attached boundary and initial conditions (see next section) has been illustrated and discussed extensively in reference [12], and hence it is omitted here.

We only mention that the finite difference method of order $O\left(h^{4}\right)$ is used to reduce the problem to that of ordinary differential equations (ODEs) with respect to $w_{i j}(t)$ and to the system of linear algebraic equations (LAEs) with respect to $F_{i j}(t)$. On each computational step, the LAE is solved through the upper relaxation method, whereas the ODEs are solved through the fourth-order Runge-Kutta method. Furthermore, the Runge principle is applied to solve the question related to stability of the applied algorithm and to the choice of an optimal computational step with respect to both spatial and time coordinates. 
4 Dissipative parametric vibrations of flexible plates

\section{Numerical investigations and results}

It should be emphasized that, contrary to the analysis of chaos in various problems of radiophysics and electronics, an investigation of complex vibrations of plates and shells is more difficult since one has to study both spatial and temporal chaoses and their interactions. Therefore, in this work, various approaches are applied to study the mentioned chaotic behaviors $[6,7,11-13,19]$.

It is obvious that in order to analyze appropriately the structure of multifrequency and chaotic vibrations, as well as the mechanisms of transitions between various vibrational regimes, one needs to analyze many potential structure characteristics. In order to obtain reliable results, it is required to use a set of various approaches, like vibrational processes measured in different points of a plate, phase, and modal portraits, FFT and power spectrum density, attractor dimension, Lyapunov exponents, Poincaré maps and pseudo-maps, bifurcation diagrams, and so on.

In what follows, we analyze a scenario of transition into a chaotic state of vibrations of a squared plate $(\lambda=a / b=1)$ made from an isotropic material $(\nu=0,3)$ subjected to the longitudinal load $P_{x}=P_{0} \sin \omega t$ and with the damping coefficient $\varepsilon=1$ of a surrounding medium.

Different combinations of the boundary conditions are applied:

$$
\begin{gathered}
w=\frac{\partial^{2} w}{\partial x^{2}}=F=\frac{\partial^{2} F}{\partial x^{2}}=0, \\
w=\frac{\partial w}{\partial x}=F=\frac{\partial^{2} F}{\partial x^{2}}=0 .
\end{gathered}
$$

Note that they change only along each side of the plate.

In a point of boundary conditions vibration, the additional condition is formulated, that is, $w_{x y}^{\prime \prime}=0$. Initial conditions read $\left.w\right|_{t=0}=\varphi_{1}(x, y),\left.w_{t}^{\prime}\right|_{t=0}=0$, and the function $\varphi_{1}(x, y)$ has been obtained through the setup method (for more details, see [7]).

Seven different types of boundary conditions, denoted as Investigations 3.1-3.7, are considered.

Investigation 3.1. The contour part $\{0 \leq x \leq 0.5, y=1\}$ satisfies condition (3.2), and the remaining contour part satisfies condition (3.1).

Investigation 3.2. One square side $\{0 \leq x \leq 1, y=1\}$ satisfies condition (3.2), whereas three other sides satisfy condition (3.1).

Investigation 3.3. Part of the boundary $\{0 \leq x \leq 1, y=1\},\{x=1,0.5 \leq y \leq 1\}$, satisfies condition (3.2), whereas the rest of the contour satisfies condition (3.1).

Investigation 3.4. Two sides of the square, that is, $\{0 \leq x \leq 1, y=1\},\{x=1,0 \leq y \leq 1\}$, satisfy condition (3.2), whereas the rest of the contour satisfies condition (3.1).

Investigation 3.5. Part of the contour, that is, $\{0 \leq x \leq 1, y=1\},\{x=1,0 \leq y \leq 1\}$, $\{0.5 \leq x \leq 1, y=0\}$, satisfies condition (3.2), and the rest of the contour satisfies condition (3.1). 


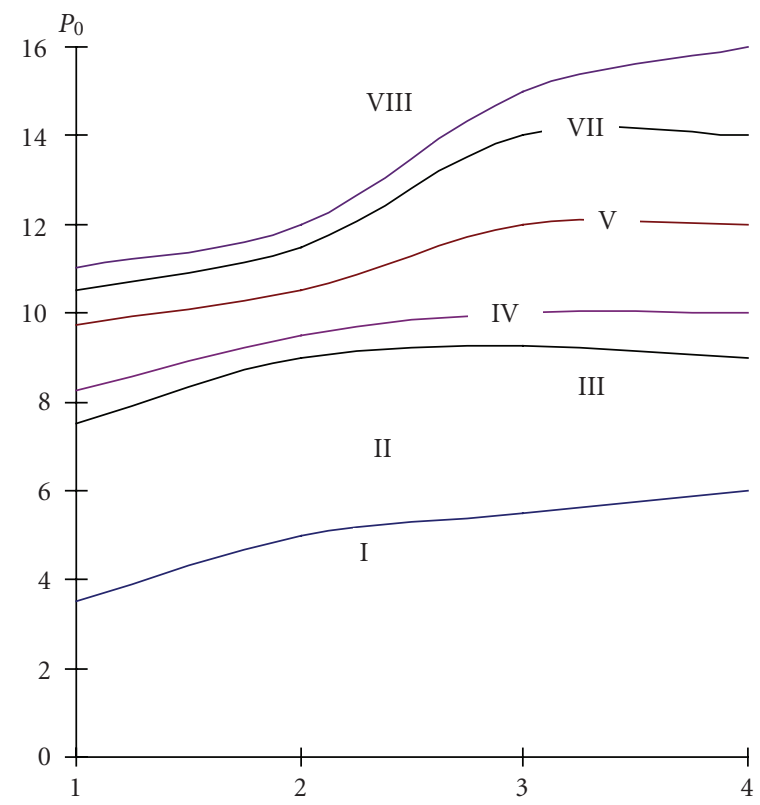

Figure 3.1. Different zones of plate vibrations (see text for more details).

Investigation 3.6. Three square sides, that is, $\{0 \leq x \leq 1, y=1\},\{x=1,0 \leq y \leq 1\},\{0 \leq$ $x \leq 1, y=0\}$, satisfy condition (3.2), whereas the rest of the contour satisfies condition (3.1).

Investigation 3.7. Part of the contour, that is, $\{x=0,0.5 \leq y \leq 1\}$, satisfies condition (3.1), and the rest of the contour satisfies condition (3.2).

For each of the stated problems, plate vibrations are analyzed through a change of the control parameter $P_{0}$ for different $\omega$, and we consider the case when $\omega$ is close to the frequency of free plate linear vibrations.

The set $\left\{P_{0}\right\}$ enables the determination of intervals of excited force, for which plate vibration has general characteristics classified as steady state, periodic, and quasiperiodic vibrations, pre-crisis, crisis, and post-crisis vibrations, as well as the pre-chaotic and chaotic vibrations. In Figure 3.1, general results of Investigations 3.1-3.4 are shown. The change of the boundary conditions is associated with vibration around stable equilibrium state (I), quasiperiodic vibrations (II), and pre-crisis (III), crisis (IV), post-crisis (V), prechaos (VI), and chaos (VII) vibrations. However, globally, the scenario of transition into a chaotic state is analogous to the scenario governed by Problem 1 (boundary conditions (3.1) are realized along the whole contour) [19]. Phase $\left(w_{t}(w)\right)$ and modal $\left(w_{x}(w)\right)$ portraits, pseudo-Poincaré maps, and FFT and $w_{\mathrm{av}}(t)$, characterizing intervals II-V, are reported in Tables 3.1, 3.2, 3.3, and 3.4. 
Table 3.1. Zone 2.

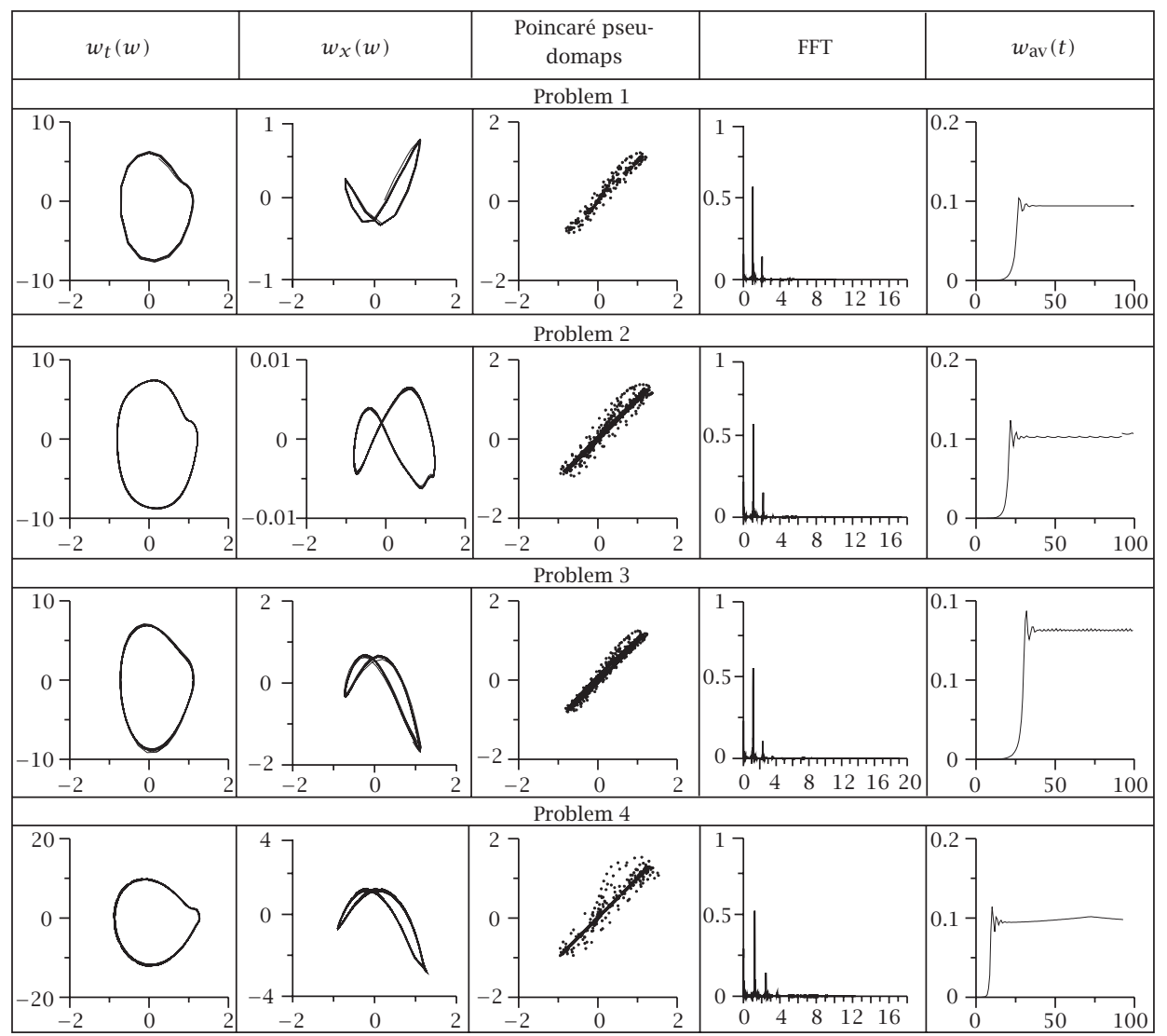

For intervals of quasiperiodic vibrations, pre-crisis, and post-crisis ones, the relations $w_{t}(w)$ and $w_{x}(w)$ are shown for a steady state time interval, whereas for crisis, they are reported in the whole investigated time interval $t \in[0,100]$.

In zone II (quasiperiodic vibrations-Table 3.1), vibrations with two fundamental frequencies occur. Other frequencies depend linearly on the two-mentioned fundamental ones. Steady state plate vibrations are realized for positive equilibrium position (see $\left.w_{\text {av }}(t)\right)$. The Poincaré pseudo-map creates an angle of $45^{\circ}$ with the axis $O_{x}$, and is represented by a straight line in the steady state regime. Phase portrait (in this interval) does not change with variation of boundary conditions (Investigations 3.1-3.4). Modal portrait, with the increase of the contour clamping beginning from 12.5\% (Investigation 3.1) up to $25 \%$, is significantly changed. The increase of clamping up to $50 \%$ (Investigation 3.4) does not essentially change the modal form portrait.

The pre-crisis state (Table 3.2) is characterized by the occurrence of a general number of frequencies depending on fundamental ones. An increase of the number of frequencies influences phase portrait shape, which is significantly more complex as compared 
Table 3.2. Pre-crisis state.

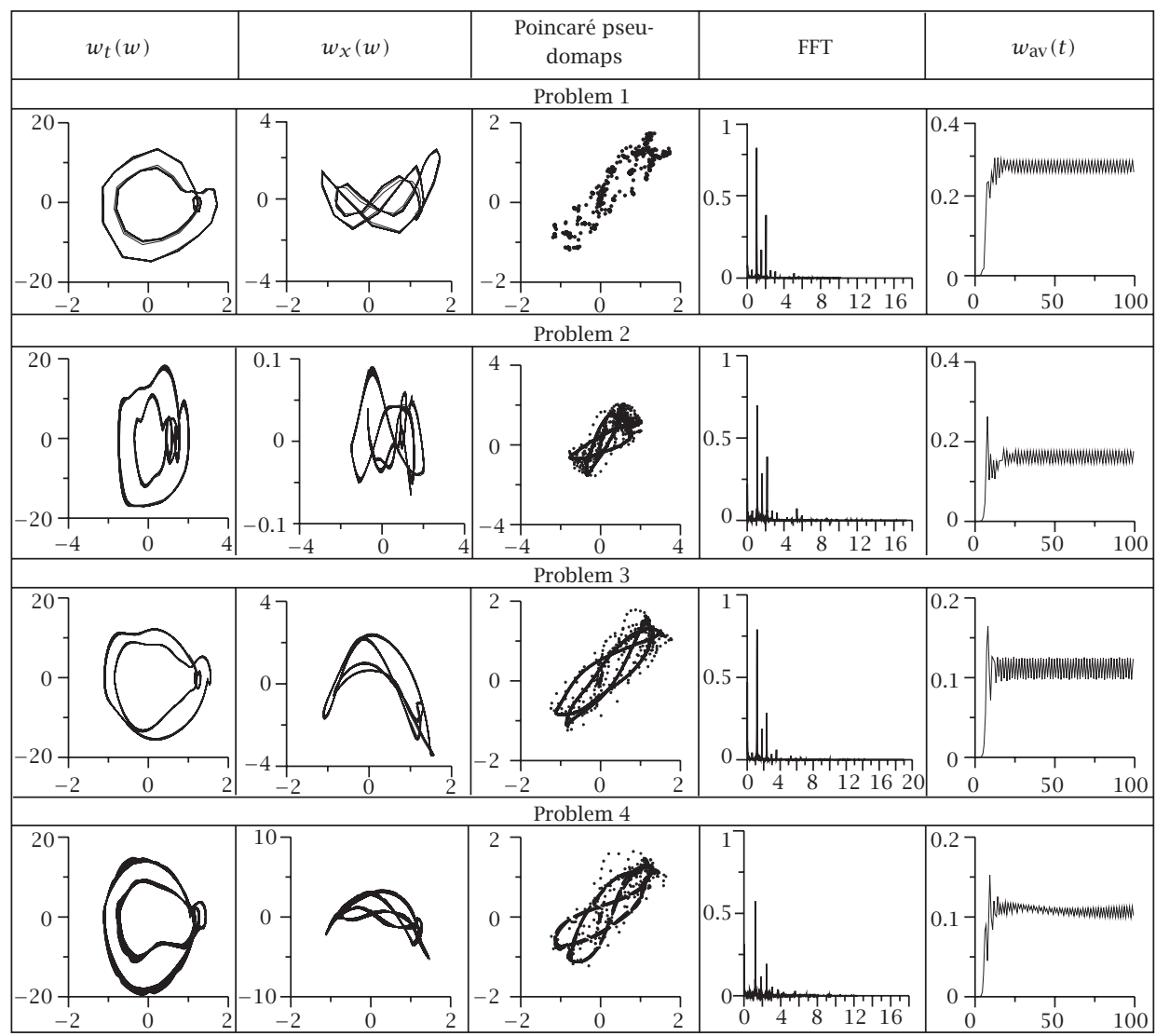

to interval II. Contrary to the interval of quasiperiodic vibrations in interval III, essential changes in the form of phase portraits are observed for each investigated change of the boundary conditions (Investigations 3.1-3.4). One may recognize the occurrence of a strange attractor in the Poincaré pseudo-map. Vibrations occur around positive equilibrium state and their amplitude increases in comparison to interval II. It is worth noting that in the pre-crisis state, a change of equilibrium state with the vibration of $P_{0}$ may occur.

A rotation of phase portrait occurs, although its form and frequency characteristics remain unchanged (Investigation 3.3).

The crisis state (Table 3.3) is characterized by the increase of independent frequencies, and power spectrum creates a broad band shape. Although the vibrational character in the whole investigated interval is chaotic, quasiperiodic windows of vibrations appear (intermittency behavior). For Investigation 3.1 at $P_{0}=8.25$ (Table 3.3), two most lengthy time intervals of quasiperiodic orbits are observed. In interval $t \in[9.8,39.2]$, vibrations take place in relation to positive equilibrium state. The phase portrait in this interval is analogous to that in the pre-crisis interval. In interval $t \in[40,68.6]$, the plate undergoes 
Table 3.3. Crisis state.

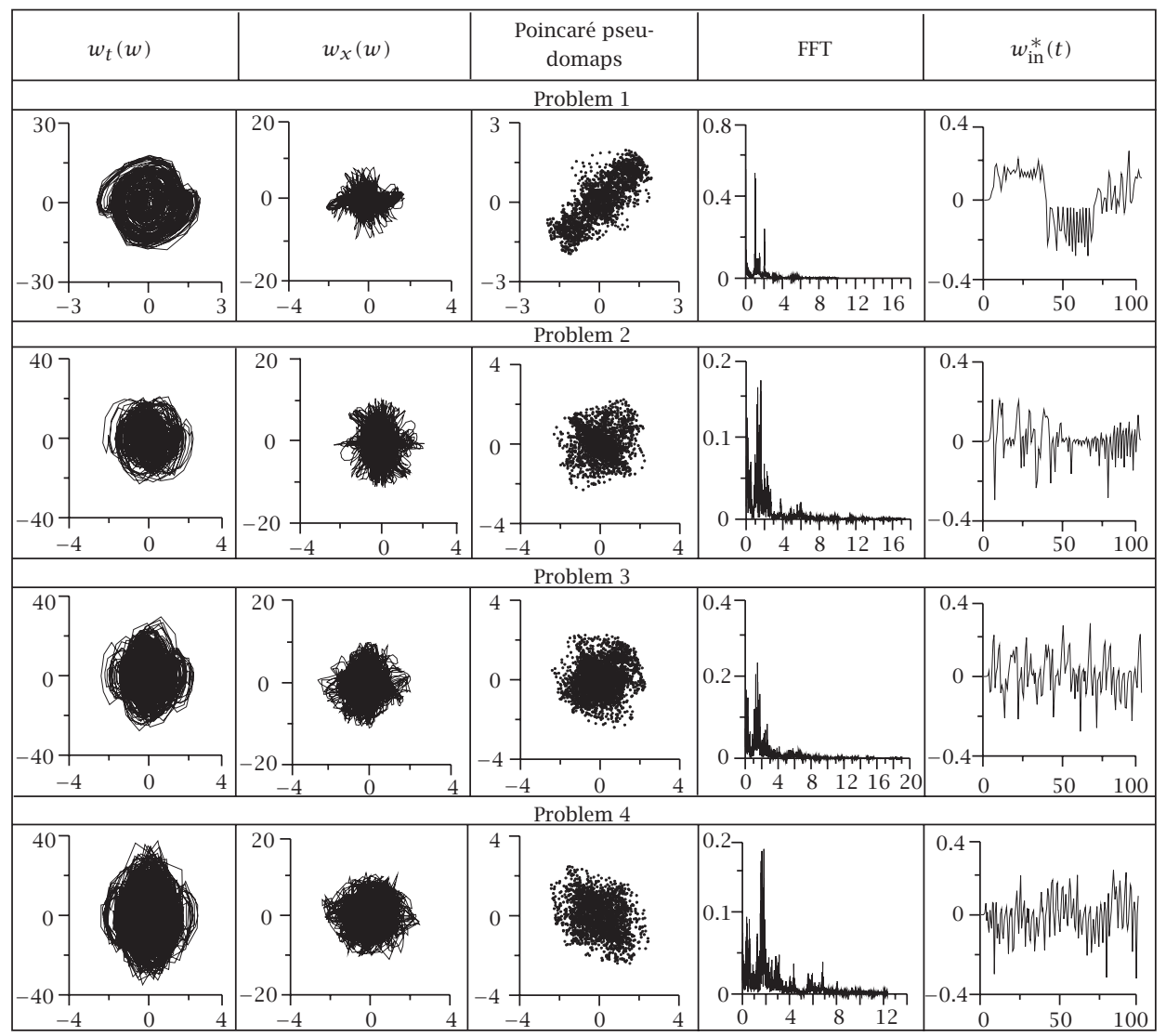

vibration around negative equilibrium state, and rotation of phase and modal portraits appear. While increasing $P_{0}$ in interval IV in the case of Investigation 3.1, the number of quasiperiodic windows as well as their duration in time are reduced. The most lengthy intervals of existence of quasiperiodic vibrations are observed for Investigations 3.2 and 3.3 for $P_{0}$ around $P_{0}=10$.

In Investigation 3.4, in the crisis state $\left(P_{0}=11\right)$, chaotic plate vibration in large time interval approaches periodic one for $t \in[73,100]$. At $P_{0}=12$ (Table 3.3), quasiperiodic windows do not appear.

In the post-crisis state (Table 3.4), vibrations occur in the "convexity-concavity" regime. The change of fundamental frequencies occurs after the crisis. The Poincaré pseudo-maps and $O_{x}$ axis create the angle of $135^{\circ}$. In the case of Investigations 3.1, 3.2, the most characteristic shape of phase portrait is reported. Increasing clamping of the contour (Investigations 3.3, 3.4), the phase portrait form is simplified. On the other hand, the modal portrait in post-crisis state with the change of boundary conditions is significantly changed. 
Table 3.4. Post-crisis state (V).

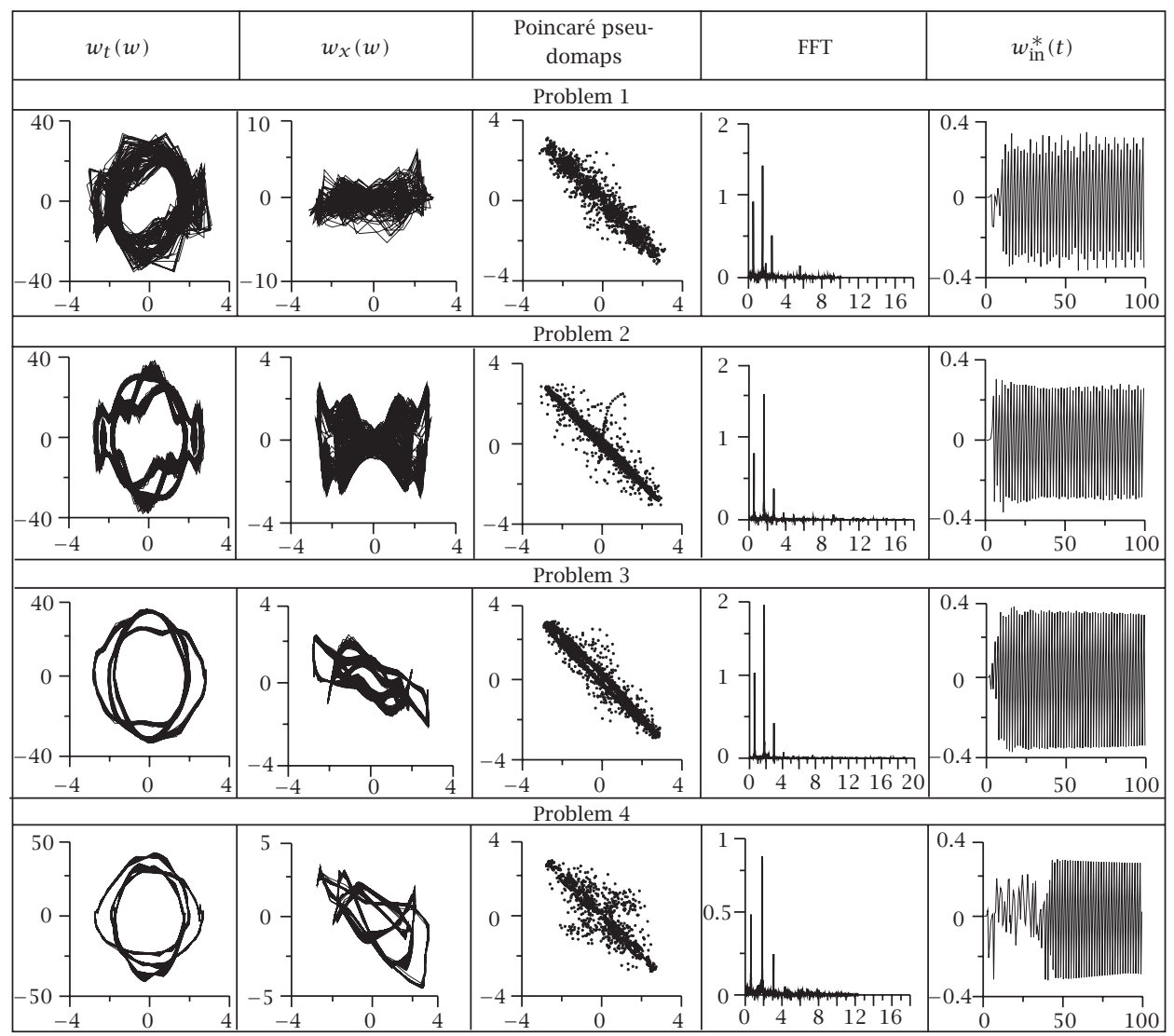

In the pre-crisis state (VI), the fundamental vibration characteristics indicate the occurrence of chaos. The number of nondependent fundamental frequencies increases, and the broad band spectrum appears. Increasing $P_{0}$ (interval VII) yields fully chaotic vibrations of plate in the considered time interval.

When considering vibrations of the plate subjected to longitudinal load $P_{x}=P_{0} \sin \omega t$ with frequencies different from the frequency of linear vibrations, it occurs that $\omega$ plays a role of the most sensitive parameter (a few crisis states are possible). Variation of $\omega$ (increase or decrease) yields occurrence of new modal forms and phase portraits.

Let us consider vibration of plate with $62.5 \%$ of its contour clamped, and with a ball type support of the remaining contour part (Investigation 3.5).

In Table 3.5, both phase and modal portraits, Poincaré pseudo-map, FFT, and $w_{\mathrm{av}}(t)$ are presented for some values of $P_{0}$.

In interval $P_{0} \in[6,10)$, plate vibrations occur with two fundamental frequencies with respect to positive (e.g., $P_{0}=9$ ) and negative equilibrium states (e.g., for $P_{0}=8$ ). The fundamental characteristics of the vibrational process of this interval correspond to the 
Table 3.5. Problem 5.

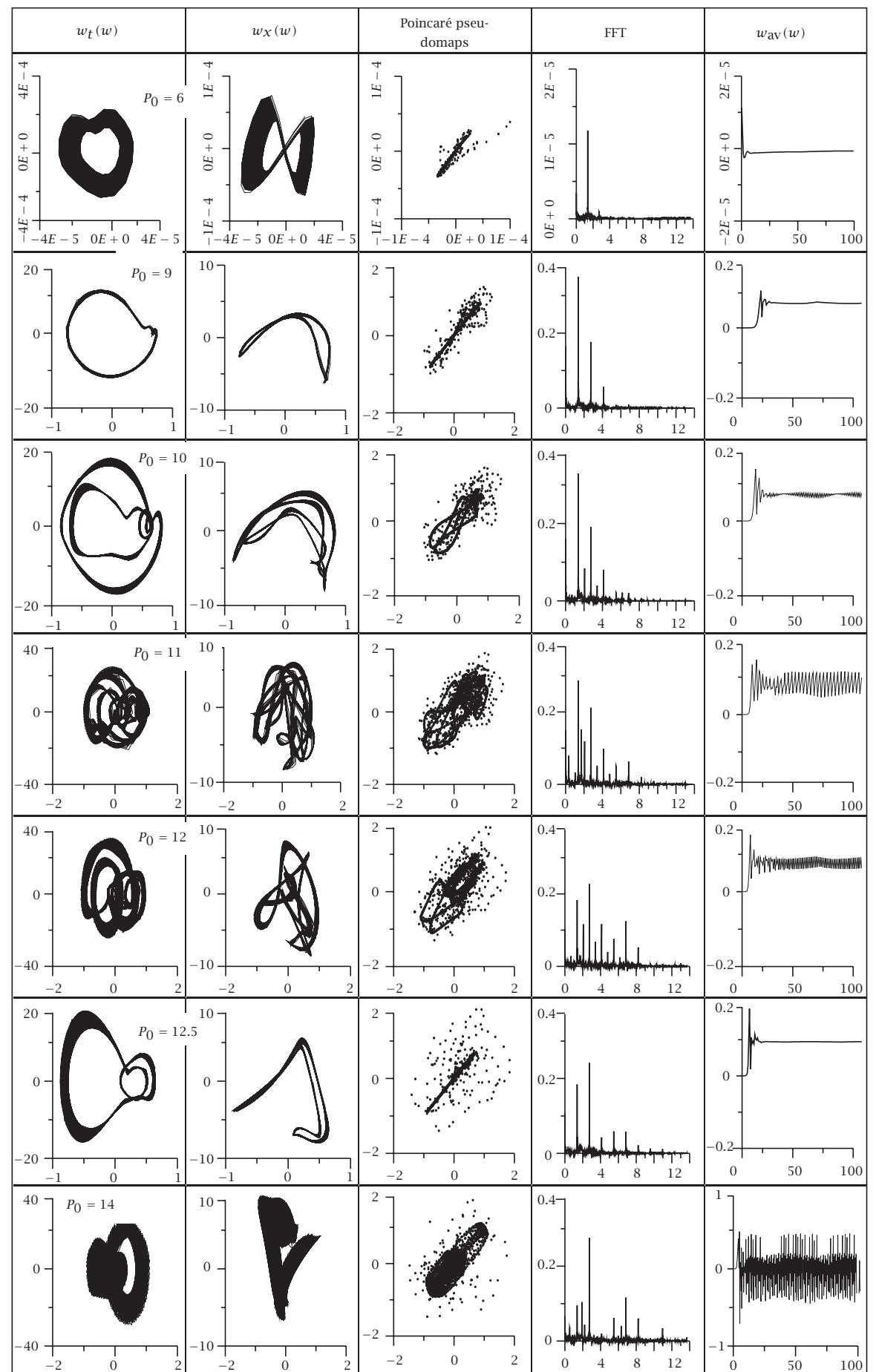


Table 3.6. Problem 6, $w_{\mathrm{av}}(t)$.

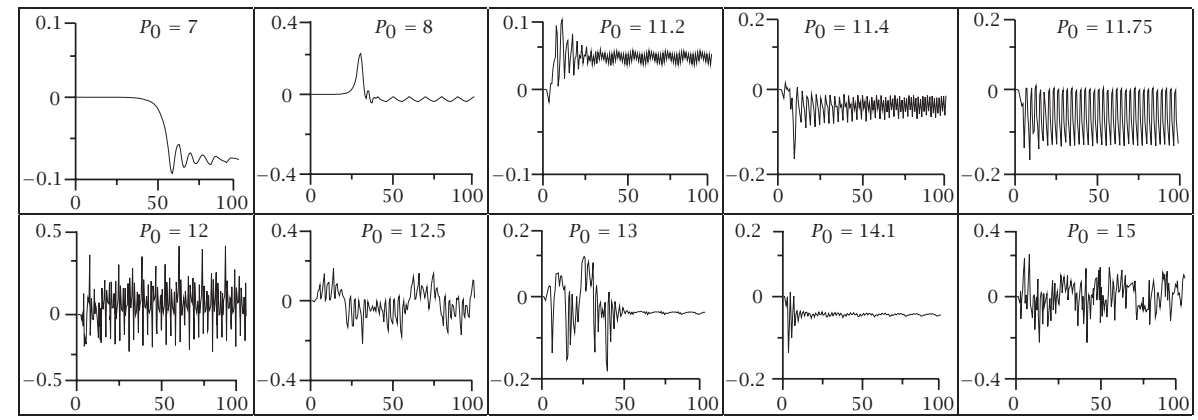

same characteristics in the interval of periodic vibrations (II) for the scenario associated with Investigations 3.1-3.4.

While increasing $P_{0}\left(P_{0} \geq 10\right)$, the vibrations include three fundamental frequencies and the increase in the number of frequencies depending on fundamental ones (Table 3.5, FFT) is analogous to the characteristics for the case of pre-crisis state (III) in the case of Investigations 3.1-3.4. In the Poincaré section, the occurrence of the strange chaotic attractor is manifested.

Increasing $P_{0}\left(P_{0} \geq 10.5\right)$ yields the extension of complexity of both modal and phase portraits. In interval $P_{0} \in[10.5,12.25)$, a change in the number of independent frequencies and frequencies dependent on them increases. Vibration takes place in relation to positive equilibrium state with the amplitude increasing in comparison to interval $P_{0} \in$ $[6,10)$ ( see $w_{\mathrm{av}}(t)$ in Table 3.5$)$.

Variations of $P_{0}\left(P_{0} \in[12.5)\right)$ force the plate to vibrate at two independent frequencies (as it was in interval $P_{0} \in[6,10)$ ), but the amplitude and the number of frequencies dependent on fundamental ones increase (Table 3.5, FFT). New structures of phase forms and modal portraits appear. Vibrations on this interval take place with respect to positive and negative equilibrium states. The change of equilibrium position causes the rotation of modal and phase portraits without variation of their forms.

Interval $P_{0} \in[14,16)$ corresponds to plate vibrations in the pre-crisis state. In the vicinity of $P_{0}=15$, frequencies are changed, and the angle of slope of Poincare pseudomap is changed. Further increase of $P_{0}$ forces the plate to vibrate in a chaotic manner.

To sum up, a transition to chaos for the considered boundary conditions and $\omega=8.6$ (close to the free plate vibration frequency) is realized through evolutionary (smooth) variation of phase and modal portraits. This scenario differs from that exhibited through Investigations 3.1-3.4 (in the latter one, there is an explicitly exhibited crisis state). In interval $P_{0} \in[10.5,12.25)$, a complexity of vibrational process increases, although the occurring vibrations are not chaotic yet.

Now (Investigation 3.6), the transition of plate vibrations into a chaotic state, when $75 \%$ of the plate contour is clamped, is analyzed (the remaining contour part is balltype supported). In order to investigate a space plate stability loss, one may analyze the characteristic $w_{\mathrm{av}}(t)$ reported in Table 3.6. It includes the whole required information on complex vibrations, jumps through equilibrium state and occurrence of convex-concave 
states. It should be emphasized that the reported characteristic yields information on crisis occurrence and chaotic vibrations as well as on intermittency processes.

In interval $P_{0} \in[6,10]$, buckling of the plate takes place in the direction opposite to initial excitation amplitude. Vibrations are realized around negative equilibrium state. For $P_{0} \geq 8$ in the considered interval of $P_{0}$ variation, firstly a buckling in the direction of initial amplitude excitation occurs, and then a successive buckling (click) takes place in the opposite direction. The increase of $P_{0} \geq 10.1\left(P_{0} \in[10.1,11.2]\right)$ causes the plate buckling (jump) into positive side equilibrium state (for the considered loading interval, we have the same equilibrium axis). For $P_{0}>11.2\left(P_{0} \in[11.2,11.75]\right)$, the plate buckling in negative direction occurs. The increase of $P_{0}$ causes a shift of neutral equilibrium state. In crisis $\left(P_{0} \in[11.75,13]\right)$, spatial chaotic state occurs, and the plate vibrates in the convexity-concavity regime. A further increase of $P_{0} \geq 13\left(P_{0} \in[13,14.4]\right)$ forces the system to buckle into negative direction and to vibrate around a new equilibrium state. Finally, the increase of $P_{0}\left(P_{0} \in[14.4, \infty]\right.$ pushes the system to vibrate chaotically.

The results obtained earlier yield the conclusion that before the crisis, four bifurcations are observed. On the other hand, after the crisis, there are two bifurcations, and after the 6th bifurcation, a spatial-temporal chaos occurs.

Analyzing the characteristics reported for some values of $P_{0}$ (Table 3.7), a conclusion is drawn that after the first bifurcation a quasiperiodic attractor appears. A simple quasiperiodic attractor is observed on the phase space and Poincaré map, that is, 1-torus is created. At increasing $P_{0}$, a series of bifurcations occurs, and "1-torus $\rightarrow 2$-torus" transition takes place, that is, a two-dimensional torus occurs. To our knowledge, the reported result belongs to the first ones observed in the theory of plates.

In Table 3.7, for $P_{0} \in[10,1,11.2]$, a smooth doubling of the initial closed curve is observed. Two invariant curves occur, which with an increase of $P_{0}$, diverge smoothly preserving ergodicity. A comparison of the mentioned map with the Poincare map for the Rayleigh-Bernard convection structure $\left(\mathrm{Ra} / \mathrm{Ra}_{c} \approx 563\right)$, as well as with the Poincaré map for the Curry-Yorke $(\varepsilon=1.465)$ model, implies similarity of the mentioned maps and existence of folds in the reported maps [4] (in Table 3.7, folds are indicated by arrows), which are characteristic for a strange attractor. In crisis, the collapse of this attractor is observed. Increasing $P_{0}$ first yields a crisis, and then a strange attractor is born, which collapses into a chaotic attractor. It is worth noting that there is a clear dependence between both temporal and spatial chaoses and the general variation of the vibrational process in time and space.

Similar to Investigation 3.5, the increase of $P_{0}$ results in complexity and increase of both phase and modal portraits (Table 3.7), which are exhibited by the increase of frequencies number. However, contrary to Investigation 3.5, for given boundary conditions, there is the interval of $P_{0} \in(11.75,13]$, where vibrations are chaotic, that is, for given boundary conditions, a crisis state is exhibited. Contrary to the scenarios reported for Investigations 3.1-3.4, in Investigation 3.6, there is no exchange of frequencies after the crisis.

Below, we consider a transition of plate vibrations into chaotic state in the case when $87.5 \%$ of the plate contour is clamped, and the remaining contour part is ball supported (Investigation 3.7). 
Table 3.7. Problem 6.

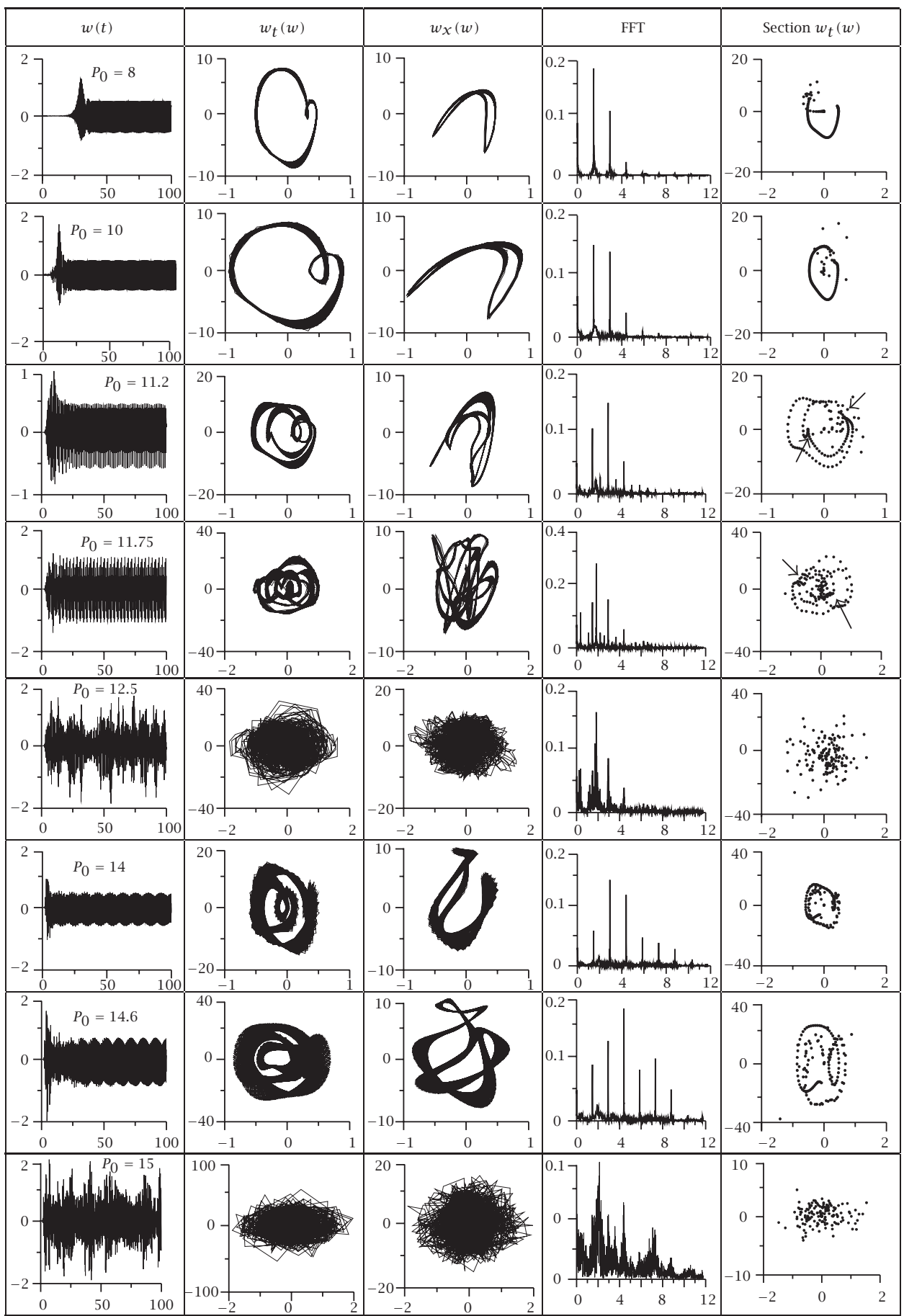


Table 3.8. Problem 7.

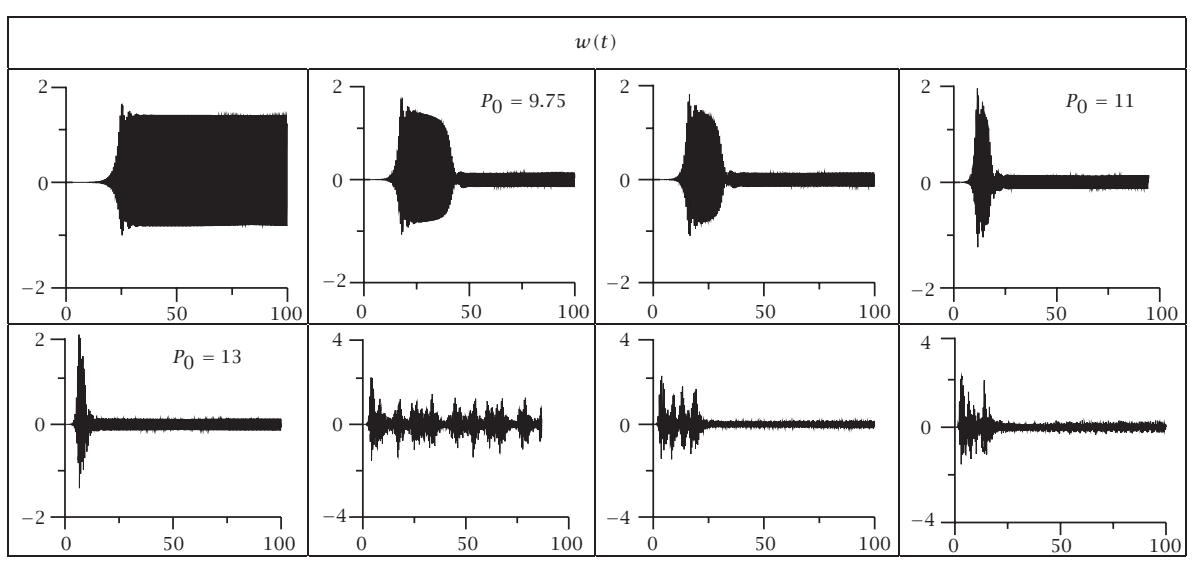

Table 3.9. Phase and modal portraits (Problem 7).

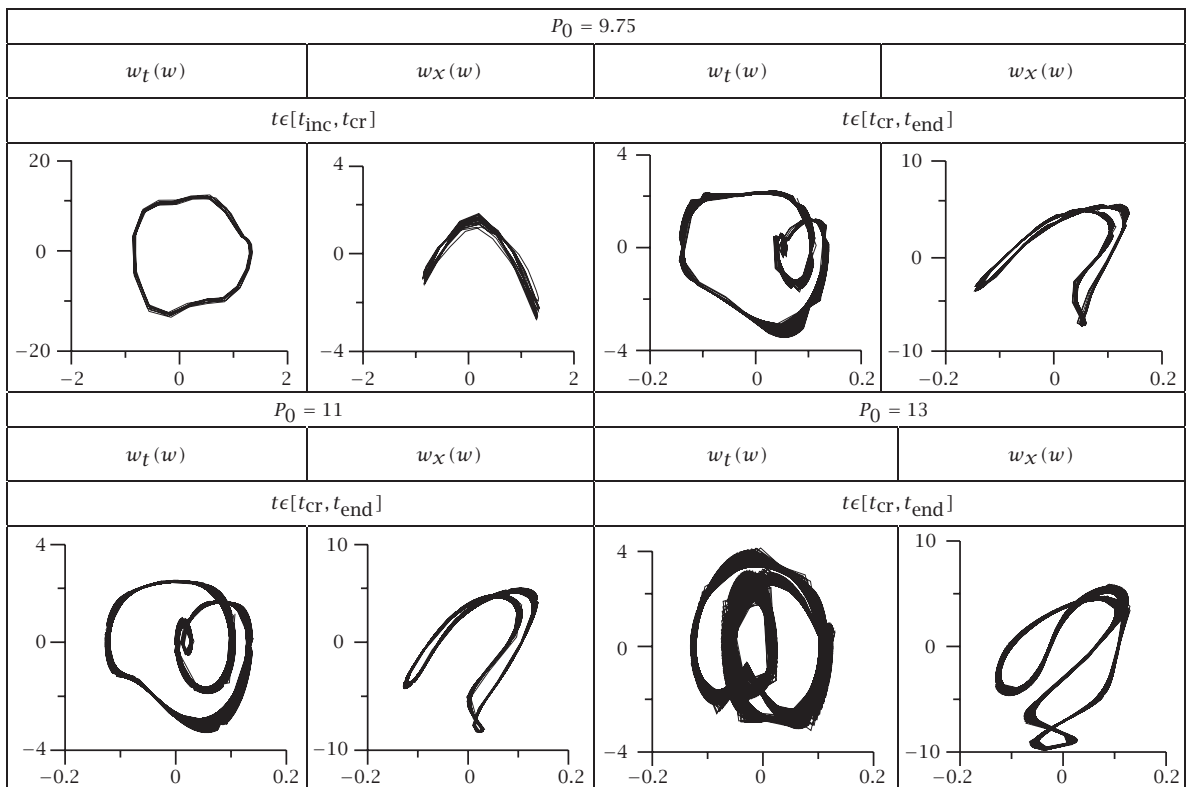

In interval $P_{0} \in(6,975)$, vibrations occur at two fundamental frequencies. The remaining frequencies that appear are linearly dependent on them. Vibrations take place with respect to positive equilibrium state. Increasing $P_{0}\left(P_{0}=9.75\right)$, a sudden change of the vibration process occurs (Table 3.8). Approaching certain $t_{\mathrm{cr}}$, the amplitude of vibrations is significantly decreased, and vibration takes place around zero equilibrium state. In the time interval from $t_{\text {inc }}$ to $t_{\mathrm{cr}}$, phase and modal portraits have the form 
analogous to $P_{0} \in(6,975)$, and beginning with $t_{\mathrm{cr}}$, phase and modal portraits take a new form (Table 3.9).

Magnitude of fundamental frequencies in intervals $t \in[0,100], t \in\left[t_{\text {inc }}, t_{\mathrm{cr}}\right], t \in\left[t_{\mathrm{cr}}\right.$, 100] do not change essentially, contrary to the change of amplitude.

Increasing $P_{0}$ causes the decrease of $t_{\text {inc }}$, and time interval between $t_{\text {inc }}$ and $t_{\mathrm{cr}}$ is reduced. In vicinity of $P_{0}=15$, frequencies exchange occurs. Vibrations take place at three fundamental frequencies, but a larger set of independent frequencies appears (broad band spectrum). Increasing $P_{0}$ shifts vibrations into a chaotic regime.

\section{Conclusions}

In this paper, regular and chaotic vibrations of flexible squared plates with boundary conditions along their contours are analyzed. The von Kármán governing equations are reduced to the hybrid system of ordinary differential and algebraic equations due to application of the finite difference method with approximation $O\left(h^{4}\right)$.

Some peculiarities of transition into chaos exhibited by vibrating plates with nonhomogeneous boundary conditions along their contours versus frequency and boundary conditions types are reported. Seven different types of boundary conditions are studied, and various dynamical states of the considered plate are analyzed. Novel scenarios for chaotic dynamical states associated with nonhomogeneous boundary conditions are illustrated and discussed.

\section{References}

[1] A. Abe, Y. Kobayashi, and G. Yamada, Two-mode response of simply supported, rectangular laminated plates, International Journal of Non-Linear Mechanics 33 (1998), no. 4, 675-690.

[2] I. V. Andrianov and J. Awrejcewicz, New trends in asymptotic approaches: Summation and interpolation methods, Applied Mechanics Reviews 54 (2001), no. 1, 69-92.

[3] I. V. Andrianov, J. Awrejcewicz, and R. G. Barantsev, Asymptotic approaches in mechanics: New parameters and procedures, Applied Mechanics Reviews 56 (2003), no. 1, 87-110.

[4] V. C. Anishchenko, Complex Oscillations in Simple Systems. Mechanisms for the Origin, Structure and Property of Dynamic Chaos in Radiophysical Systems, Nauka, Moscow, 1990.

[5] J. Awrejcewicz, I. V. Andrianov, and L. I. Manevitch, Asymptotic Approaches in Nonlinear Dynamics. New Trends and Applications, Springer Series in Synergetics, Springer, Berlin, 1998.

[6] J. Awrejcewicz and A. V. Krysko, Analysis of complex parametric vibrations of plates and shells using Bubnov-Galerkin approach, Archive of Applied Mechanics 73 (2003), no. 7, 495-504.

[7] J. Awrejcewicz and V. A. Krysko, Nonclassical Thermoelastic Problems in Nonlinear Dynamics of Shells, Scientific Computation, Springer, Berlin, 2003.

[8] Some problems of analysis and optimization of plates and shells, Journal of Sound and Vibration 264 (2003), no. 2, 343-376.

[9] __ Wavelets-based analysis of parametric vibrations of flexible plates, International Applied Mechanics 39 (2003), no. 9, 997-1028.

[10] On the vibration of the Euler-Bernoulli beam with clamped ends deflection constraints, International Journal of Bifurcation and Chaos in Applied Sciences and Engineering 15 (2005), no. 6, 1867-1878, Special issue.

[11] J. Awrejcewicz, V. A. Krysko, and A. V. Krysko, Regular and chaotic behaviour of flexible plates, Proceedings of the Third International Conference on Thin-Walled Structures (Carcow, 2001) (J. Zaraś, K. Kowal-Michalska, and J. Rhodes, eds.), Elsevier Science, Amesterdam, 2001, pp. 349-356. 
[12] Spatio-temporal chaos and solitons exhibited by von Kármán model, International Journal of Bifurcation and Chaos in Applied Sciences and Engineering 12 (2002), no. 7, 1465-1513.

[13] Complex parametric vibrations of flexible rectangular plates, Meccanica 39 (2004), no. 3, 221-244.

[14] J. Awrejcewicz, V. A. Krysko, and G. G. Narkaitis, Bifurcations of a thin plate-strip excited transversally and axially, Nonlinear Dynamics 32 (2003), no. 2, 187-209.

[15] V. A. Krysko, J. Awrejcewicz, and V. M. Bruk, On the solution of a coupled thermo-mechanical problem for non-homogeneous Timoshenko-type shells, Journal of Mathematical Analysis and Applications 273 (2002), no. 2, 409-416.

[16] , The existence and uniqueness of solution of one coupled plate thermomechanics problem, Journal of Applied Analysis 8 (2002), no. 1, 129-139.

[17] V. A. Krysko, J. Awrejcewicz, A. N. Kutsemako, and K. Broughan, Interaction between flexible shells (plates) and a moving lumped body, Communications in Nonlinear Science and Numerical Simulation 11 (2006), no. 1, 13-43.

[18] V. A. Krysko, J. Awrejcewicz, and G. G. Narkaitis, Nonlinear vibration and characteristics of flexible plate-strips with non-symmetric boundary conditions, Communications in Nonlinear Science and Numerical Simulation 11 (2006), no. 1, 95-124.

[19] V. A. Krysko, T. V. Vakhlaeva, and A. V. Krysko, Dissipative vibrations of flexible plates and their transition into spatial-temporal chaos due to harmonic longitudinal excitation, Nonlinear Dynamics of Mechanical and Biological Systems, Saratov University Press, Saratov, 2000.

[20] I. Lasiecka, Finite dimensionality and compactness of attractors for von Kármán equations with nonlinear dissipation, NoDEA. Nonlinear Differential Equations and Applications 6 (1999), no. 4, 437-472.

[21] T. A. Nayfeh and A. F. Vakakis, Subharmonic travelling waves in a geometrically nonlinear circular plate, International Journal of Non-Linear Mechanics 29 (1994), no. 2, 233-245.

[22] W. Zhang, Z. Liu, and P. Yu, Global dynamics of a parametrically and externally excited thin plate, Nonlinear Dynamics 24 (2001), no. 3, 245-268.

J. Awrejcewicz: Department of Automatics and Biomechanics, Technical University of Lódź, 1/15 Stefanowski Street, 90-924 Łódź, Poland

E-mail address: awrejcew@p.lodz.pl

V. A. Krysko: Mechanics and Mathematics Department, Saratov State University,

B. Sadovaya, 96 ${ }^{a}$, fl. 77, 410054 Saratov, Russia

E-mail address: tak@san.ru

T. Moldenkova: Mechanics and Mathematics Department, Saratov State University,

B. Sadovaya, 96 ${ }^{a}$, fl. 77, 410054 Saratov, Russia

E-mail address: tak@san.ru 


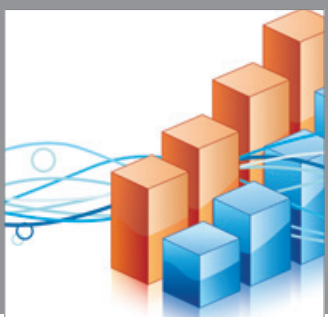

Advances in

Operations Research

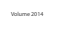

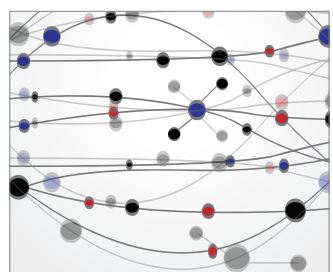

\section{The Scientific} World Journal
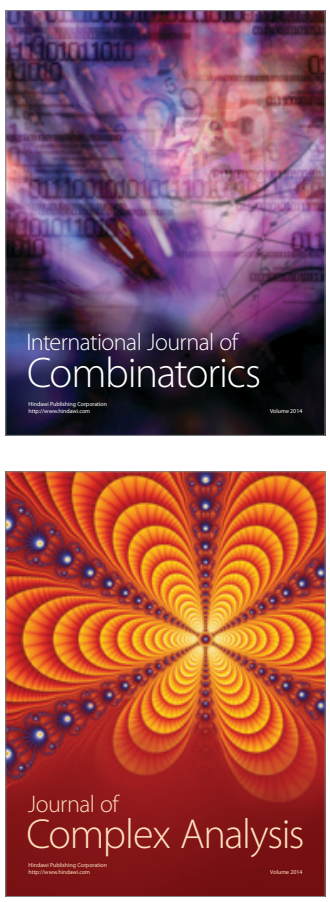

International Journal of

Mathematics and

Mathematical

Sciences
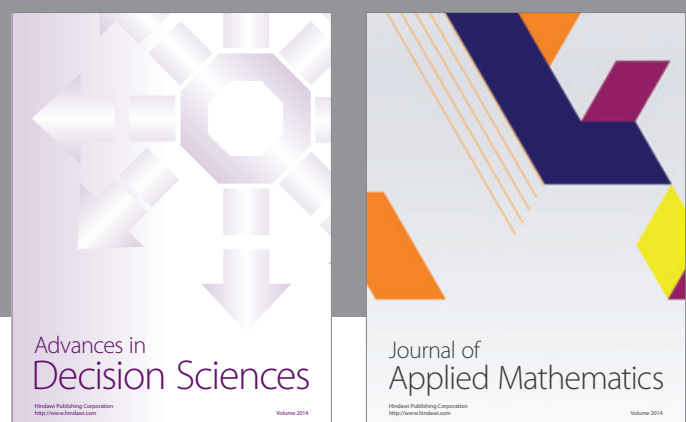

Journal of

Applied Mathematics
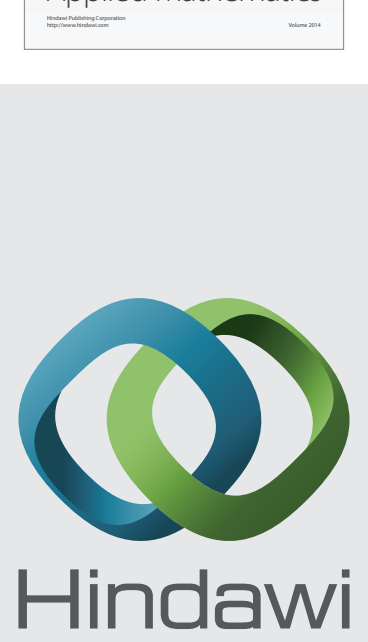

Submit your manuscripts at http://www.hindawi.com
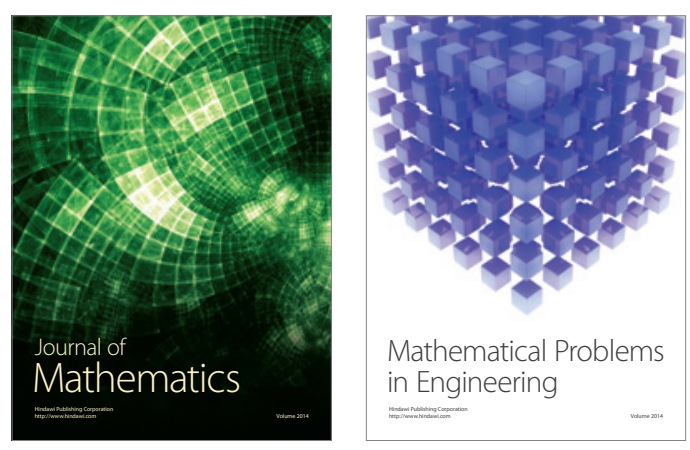

Mathematical Problems in Engineering
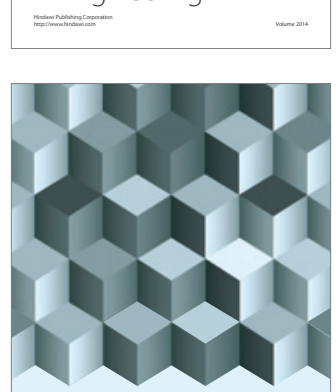

Journal of

Function Spaces
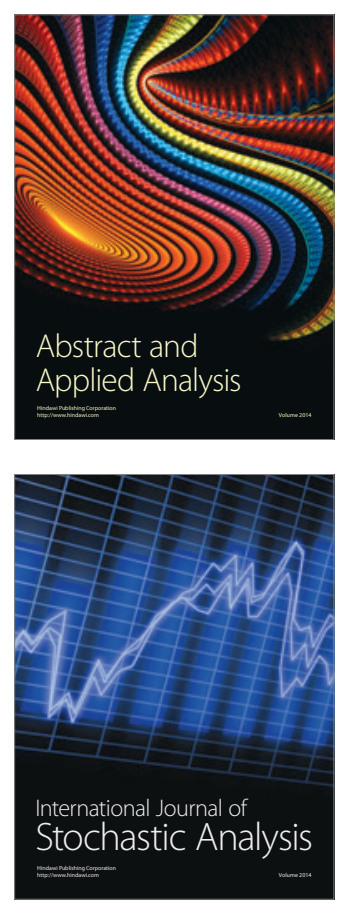

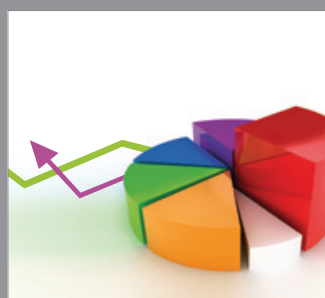

ournal of

Probability and Statistics

Promensencen
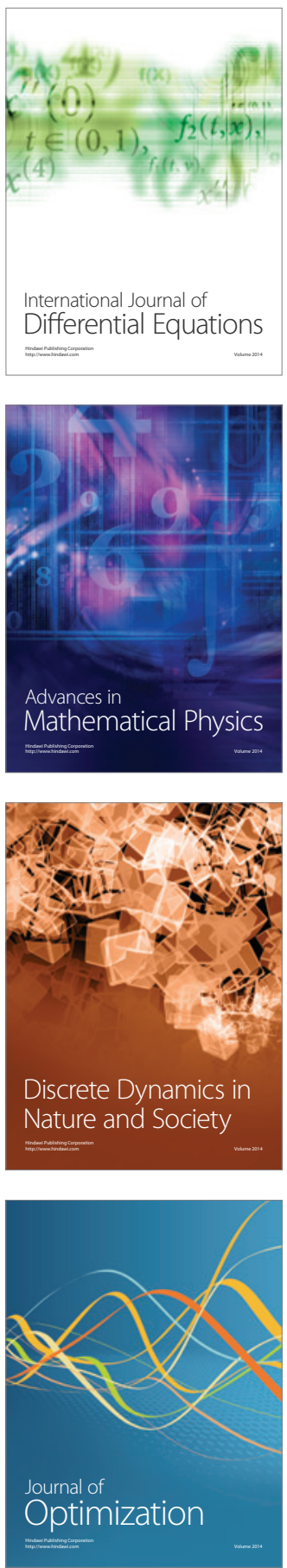